\title{
The synergistic apoptotic effects of thiophenfurin, an inosine monophosphate dehydrogenase inhibitor, in combination with retinoids in HL60 cells
}

\author{
MARIA MELI $^{1}$, MANLIO TOLOMEO $^{2}$, MARIO GRIFANTINI $^{4}$, PALMARISA FRANCHETTI ${ }^{4}$, \\ LOREDANA CAPPELLACCI ${ }^{4}$, DANIELE SIMONI ${ }^{5}$, FRANCESCO P. INVIDIATA ${ }^{3}$, \\ STEFANIA AIELLO ${ }^{3}$ and LUISA DUSONCHET ${ }^{1}$
}

\author{
${ }^{1}$ Dipartimento di Scienze Farmacologiche, Università di Palermo; ${ }^{2}$ Cattedra di Ematologia e Servizio AIDS, \\ Azienda Ospedaliera Universitaria Policlinico di Palermo, via del Vespro 129, 90127 Palermo; \\ ${ }^{3}$ Dipartimento Farmacochimico, Tossicologico e Biologico, Università di Palermo, via Archirafi 32, \\ 90123 Palermo; ${ }^{4}$ Dipartimento di Scienze Chimiche, Università di Camerino, via \\ Sant' Agostino 1, 62032 Camerino; ${ }^{5}$ Dipartimento di Scienze Farmaceutiche, \\ Università di Ferrara, via Fossato di Mortasa 17-19, 44100 Ferrara, Italy
}

Received June 14, 2006; Accepted August 1, 2006

\begin{abstract}
New effective cytotoxic agents and combinations are urgently needed in cancer treatment. The enzyme inosine monophosphate dehydrogenase is a potentially useful target for drug development, since its activity has been shown to be amplified in malignant cells. Thiophenfurin, an inhibitor of the enzyme synthesized by us, is endowed with a significant apoptotic activity in promyelocytic leukaemia HL60 cells. Since retinoids were successfully employed in the treatment of patients with leukaemia, demonstrating significant differentiation-inducing and apoptotic effects, we carried out this study to evaluate the effects of the combination of thiophenfurin and several retinoid molecules, acting in different phases of the cell cycle in vitro. The results show that thiophenfurin is capable of eliciting significant $\mathrm{S}$ phasespecific antiproliferative effects in different sensitive and resistant cell lines with the $\mathrm{IC}_{50} \mathrm{~s}$ ranging from 6.7 to $26 \mu \mathrm{M}$. When HL60 cells were treated with thiophenfurin in combination with retinoids, the effects on cell growth were additive or synergistic, depending on the kind of retinoid used and the sequence of treatment: In particular, we observed additive effects when the cells were exposed to thiophenfurin and all-transretinoic acid either simultaneously or sequentially. Instead, when the new heterocyclic retinoid isoxazole benzoic
\end{abstract}

Correspondence to: Professor Luisa Dusonchet, Dipartimento di Scienze Farmacologiche, Università di Palermo, via del Vespro 129, 90127 Palermo, Italy

E-mail:dusonc@unipa.it

Key words: apoptotic agents, antitumor agents, inosine monophosphate dehydrogenase inhibitors, tiazofurin analogues, retinoids acid was used, synergism was obtained in the cells treated sequentially. The combination of thiophenfurin and isoxazole benzoic acid determined synergistic apoptotic effects through a mitochondrion-dependent mechanism, suggesting the possible usefulness of this combination in the treatment of leukaemia.

\section{Introduction}

The inhibitors of the enzyme inosine monophosphate dehydrogenase (IMPDH) are a relatively new class of anticancer, antiviral and immunosuppressive agents. The lead compound tiazofurin (TR) demonstrated a positive antitumor activity in patients with acute myeloid leukaemia and in the blastic crisis of chronic myeloid leukaemia with a relatively tolerable toxicity profile (1). These compounds are endowed with a rather unique mechanism of action. After being metabolized to TAD (thiazole-4-carboxamide adenine dinucleotide), they impair GTP synthesis by the de novo pathway, interfering with the functions of a number of GTP-dependent proteins including c-Ki-ras, c-Ha-ras, c-myc and phospholipase $\mathrm{C}$ which are known to possess a definite role in the progression of the cell cycle and tumor cell survival $(2,3)$. Furthermore, GTP depletion may clearly interfere with DNA synthesis in rapidly cycling tumor cells. Indeed, unlike other compounds of the group, tiazofurin induces a typical arrest of the cells in the $\mathrm{S}$ phase of the cell cycle (4), as well as cell differentiation and induction of apoptotic cell death in several tumor models in vitro $(5,6)$. However, the mechanisms by which the apoptotic process is triggered by these compounds have not received significant attention to date. Overall, the above observations suggest the possibility of successfully combining these compounds with drugs possessing different mechanisms of action and possibly capable of killing cells in separate phases of the cell cycle. In this context, recent studies have been addressed to identify new compounds specific for the G1 phase in order to affect the high proportion of tumor cells 
that are not in the proliferative compartment and that are often responsible for tumor relapses as well as the emergence of drug resistance. The large class of retinoid compounds fits these requirements well. The differentiating agent all-transretinoic acid (ATRA) has attracted a great deal of interest due to its ability to induce high response rates in the treatment of acute promyelocytic leukaemia (APL) without the toxicity of the classic chemotherapeutic drugs and now represents the standard of treatment for this disease when employed in combination with chemotherapy (7). However, the benefits of ATRA are somewhat restricted to APL due to the presence of the specific chromosomal translocation (t15;17) involving the retinoid receptor $\mathrm{RAR}_{a}$, as this compound has little apoptotic activity when tested in vitro in different tumor systems (8). Nevertheless, new retinoid-like compounds are emerging that can overcome these limitations, exhibiting a high apoptotic-inducing activity in several tumor types, while only a few of them retain the ability to bind to the different classes of specific receptors (9-11). In a previous study, the heterocycle-containing retinoid, isoxazole benzoic acid (MC18), showed both cytotoxic and receptor transactivation activity in sensitive as well as resistant leukaemia cells (12).

Our group synthesized a series of analogs of tiazofurin with the aim of improving the antitumor and apoptotic activity of the molecule (13-14). The C-nucleoside thiophenfurin (5-3-D-ribofuranosylthiophene-3-carboxamide, TF) could possibly fulfil these characteristics because it showed significant apoptotic effects in preliminary experiments. On this basis, we studied the combination of thiophenfurin with ATRA as well as with the cytotoxic retinoid-related molecule MC18.

\section{Materials and methods}

Chemicals and reagents. Thiophenfurin was synthesized as described previously (13). Tiazofurin was kindly supplied by M. Grifantini. ATRA was purchased from Sigma S.p.a. MC18 corresponds to isoxazole benzoic acid (4-[5-(5,6,7,8tetrahydro-5,5,8,8-tetramethylnaphthalen-2-yl)isoxazol-3yl]benzoic acid) and was synthesized as described previously (12). The caspase 8 inhibitor Z-IETD-FMK (Z-Ile-Glu-ThrAsp-fluoromethylketone), the caspase 9 inhibitor Z-LEHDFMK [Z-Leu-Glu (OMe)-His-Asp (OMe)-fluoromethylketone], the caspase 3 inhibitor Z-DEVD-FMK [Z-Asp (OMe)-Glu (OMe)-Val-Asp (OMe)-fluoromethylketone] and the pancaspase inhibitor Z-VAD-FMK (benzyloxycarbonyl-Val-Ala-Asp-fluoromethylketone) were purchased from Alexis Biochemicals (Laufelfingen, Switzerland). The anti-Fas monoclonal antibody (mAb) ZB4 was purchased from Upstate Biotechnology (Lake Placid NY, USA). 3,3'Dihexyloxacarbocyanine iodide $\left(\mathrm{DiOC}_{6}\right)$ was supplied by Calbiochem (San Diego, CA, USA). All the other reagents were of analytical grade.

Cell culture and treatment. The human acute promyelocytic cell line HL60 was employed in the study. Since the IMPDH inhibitors were reported to inhibit $\mathrm{T}$ lymphocyte proliferation, they were also used on the human T-cell lymphoma cell line HuT78 (15). Both cell lines do not express p53 and the Pglycoprotein; furthermore, HL60 cells do not express the fusion gene PML-RAR . The multidrug resistant variant of HL60, HL60R, and a gemcitabine-resistant clone of HuT78 (HuT78R2) were also employed. HL60R cells express Pglycoprotein and are about 300 -fold resistant to the cytotoxic effects of the anticancer drug doxorubicin; in addition, this cell line showed a high degree of resistance to apoptosis induction by different chemically unrelated agents. HuT78R2 cells were selected by us in vitro by exposure to cytotoxic concentrations of gemcitabine and are about 1000fold resistant to this drug due to very reduced levels of the enzyme deoxycitidine kinase. Cells were cultured in RPMI1640 medium (Gibco, Grand Island, NY, USA) supplemented with $10 \%$ (v/v) heat-inactivated FCS (Gibco), $100 \mathrm{U} / \mathrm{ml}$ penicillin (Gibco), $100 \mu \mathrm{g} / \mathrm{ml}$ streptomycin (Gibco) and $2 \mathrm{mM}$ of L-glutamine (Gibco) at $37^{\circ} \mathrm{C}$ in a humidified $5 \% \mathrm{CO}_{2}$ atmosphere.

Cell viability and apoptosis. Cytotoxicity was assessed by the trypan blue dye exclusion test. In brief, the cells were seeded at a density of $0.2 \times 10^{6}$ cells $/ \mathrm{ml}$ in 24 -well plates and, after $24-\mathrm{h}$ incubation in a drug-free medium, different concentrations of drugs were added. After 24- or 48-h exposure, aliquots of the cells were harvested, stained with trypan blue and counted in a haemocytometer. All the samples were measured in duplicate in at least three independent experiments. The resistance index was calculated as the ratio between the $\mathrm{IC}_{50}$ (inhibitory concentration 50\%) in the experimental sample and that in the parental sensitive cell line. For the determination of the interactions between the drugs the fractional product method of Webb was applied (16). The predicted values (c) were calculated according to the equation $\mathrm{c}=\mathrm{a} \times \mathrm{b} / 100$ where $\mathrm{a}$ and $\mathrm{b}$ indicate cell survival values with single agents. Hence, the combination index (C.I.) was calculated as the ratio between the survival observed with the combinations and the predicted values. The equations C.I.=1, C.I. $<1$ and C.I. $>1$ indicate additive effects, synergism and antagonism, respectively.

Apoptosis was evaluated by fluorescence microscopy according to the method of Duke and Cohen (17). After treatment with different concentrations of the drugs for 24-48 h, the cells were centrifuged and the pellet was resuspended in $25 \mu 1$ of a dye mixture containing acridine orange and ethidium bromide. Live and apoptotic cells were identified by fluorescence microscopy. The inhibitory anti-Fas mAb or the caspase inhibitors were added $1 \mathrm{~h}$ before the drug treatment. Alternatively, apoptosis was evaluated by flow cytometry as the percentage of hypodiploid nuclei accumulated in the sub- $\mathrm{G}_{0}-\mathrm{G}_{1}$ peak after labeling with propidium iodide (18).

Cell cycle analysis. The effects of the compounds on the cell cycle were studied by flow cytometry. Briefly, the cells were washed once in ice-cold PBS and resuspended at $1 \times 10^{6} / \mathrm{ml}$ in a hypotonic fluorochrome solution containing $50 \mu \mathrm{g} / \mathrm{ml}$ propidium iodide (Sigma) in $0.1 \%$ sodium citrate plus $0.03 \%$ (v/v) Nonidet P-40 (Sigma). After 30-min incubation in this solution, the samples were filtered through a nylon cloth, $40 \mu \mathrm{m}$ mesh, and their fluorescence was analyzed as singleparameter frequency histograms by using a FACSort (Becton-Dickinson, Mountain View, CA, USA). A minimum 
Table I. The cytotoxic and apoptotic activity of TR and TF in the sensitive or resistant cell lines.

\begin{tabular}{lcclll}
\hline Cell line & \multicolumn{2}{c}{$\mathrm{TR}(\mu \mathrm{M})$} & & \multicolumn{2}{c}{$\mathrm{TF}(\mu \mathrm{M})$} \\
\cline { 2 - 3 } \cline { 6 - 6 } & $\mathrm{IC}_{50}{ }^{\mathrm{a}}$ & $\mathrm{AC}_{50}{ }^{\mathrm{b}}$ & & $\mathrm{IC}_{50}$ & $\mathrm{AC}_{50}$ \\
\hline HL60 & $9.9 \pm 3.7$ & $>100$ & & $6.7 \pm 2.5$ & $34.1 \pm 15.1$ \\
HL60R & $9.6 \pm 3$ & $>100$ & & $9.6 \pm 2.8$ & $>100$ \\
HuT78 & $40.7 \pm 9.2$ & $>100$ & & $26 \pm 8$ & $50 \pm 15$ \\
HuT78R2 & $36.8 \pm 11$ & $>100$ & & $15 \pm 8.5$ & $50 \pm 12.5$ \\
\hline
\end{tabular}

The cells were exposed to the drugs for $48 \mathrm{~h}$. Results are the mean \pm S.D. of at least three separate experiments. ${ }^{\mathrm{a}} \mathrm{IC}_{50}$ indicates the drug concentrations inhibiting cell growth by $50 \%$. ${ }^{b} \mathrm{AC}_{50}$ indicates the drug concentrations inducing apoptosis in $50 \%$ of the cells.

of 10,000 events for each sample were collected in the list mode. The distribution of cells in the cell cycle was analyzed with the ModFit LT program (Verity Software House, Inc.).

Assessment of mitochondrial membrane potential. The mitochondrial membrane potential $(\Delta \Psi)$ was measured by flow cytometry after staining the cells with the cationic lipophilic fluorochrome $\mathrm{DiOC}_{6}$ (3,3'-dihexyloxacarbocyanine iodide). In brief, the cells were harvested, washed once in PBS and incubated with $\mathrm{DiOC}_{6}(40 \mathrm{nM})$ for $15 \mathrm{~min}$ at $37^{\circ} \mathrm{C}$. The analysis was carried out by flow cytometry and the percentage of cells exhibiting low levels of $\mathrm{DiOC}_{6}$, reflecting a loss of mitochondrial membrane potential, was determined.

\section{Results}

Cytotoxic and apoptotic effects of TF. The ability of TF to kill tumor cells was assayed in vitro in comparison with the reference molecule TR in HL60 and HuT78 cells. As shown in Table I, the compound is endowed with a significant cytotoxic activity against HL60 cells with an $\mathrm{IC}_{50}$ of $6.7 \mu \mathrm{M}$, slightly lower than that exhibited by TR. Furthermore, while TR-induced apoptosis was almost negligible in HL60 cells even at $100 \mu \mathrm{M}$, TF was actively apoptotic with an $\mathrm{AC}_{50}$ of $34.1 \mu \mathrm{M}$. Likewise, TF was more cytotoxic than TR in HuT78 cells, although, on the whole, this lymphatic tumor cell line was less sensitive to both IMPDH inhibitors. To evaluate the effectiveness of TF against drug-resistant tumors, the multidrug-resistant HL60R cell line and the HuT78R2 cells were also treated with increasing concentrations of the drugs. The IMPDH inhibitors showed cytotoxic effects in resistant cells that were at least comparable with those observed in the parental cell lines; however, HL60R cells were highly resistant to apoptosis induction by both compounds.

Effect of TF on cell cycle distribution of HL60 cells. To examine the influence of TF on the cell cycle distribution in HL60 cells, the cells were exposed for various times to fully cytotoxic concentrations of the drug or the reference compound TR and evaluated by flow cytometry after incubation with propidium iodide as described in Materials and methods. As shown in Fig. 1, TF, as well as TR, showed a significant $\mathrm{S}$ phase-specific effect by blocking $62 \%$ of the cells in this phase of the cell cycle after 24-h incubation with the complete disappearance of the latest $\mathrm{G} 2$ and $\mathrm{M}$ phase cell fractions. This phase $\mathrm{S}$ specificity suggested the feasibility of drug combinations with G1-specific molecules such as retinoids.

Influence of the combination of TF and ATRA on the growth of HL60 cells. To study the effect of drug combinations, HL60 cells were exposed for $24 \mathrm{~h}$ to $20 \mu \mathrm{M}$ ATRA alone or in combination with TF $(10 \mu \mathrm{M})$ or TR $(10 \mu \mathrm{M})$. As shown in Table II, the cytotoxic effect of ATRA employed alone was $18 \%$, whereas it was $43 \%$ and $41 \%$ for TF and TR, respectively. Instead, when the cells were exposed to the drugs in combination, the cytotoxic effect observed was similar to the sum of the effects of each compound employed alone. Furthermore, in order to evaluate whether this result was influenced by the sequence of the exposure to the drugs, the cells were exposed to one drug for $24 \mathrm{~h}$, washed in a drug-free medium and were then exposed to the second drug for a further $24 \mathrm{~h}$. The results showed that the combination of TR followed by ATRA was less than additive whereas the combinations of ATRA with TF were fully additive, irrespective of the sequence of exposure.

Cytotoxic effects of the combination of $T F$ with new retinoid molecules. Although the above results suggest that the combinations of ATRA with TF may be suitable for further drug development studies due to additive cytotoxic effects, we wanted to determine whether combining this molecule with more potent and cytotoxic retinoids can further increase the antitumor effects of the combinations. Therefore, we studied the effects of TF in combination with the retinoid analog MC18 in HL60 cells. We have previously shown that
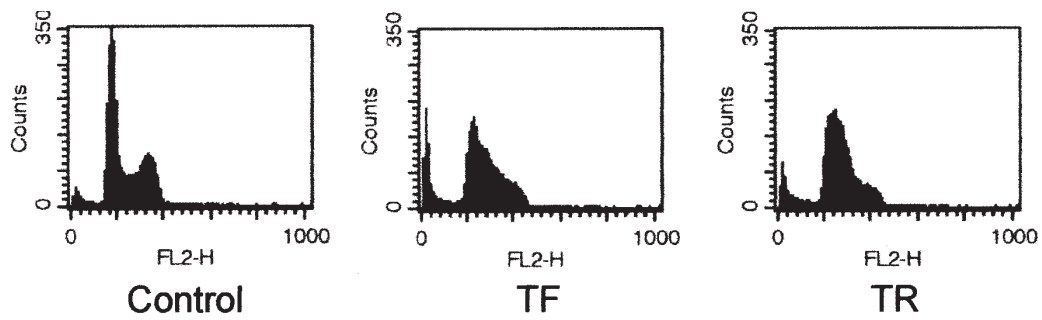

Figure 1 . The cell cycle distribution of HL60 cells treated with the IMPDH inhibitors. The cells were exposed to $20 \mu \mathrm{M}$ TR or $10 \mu \mathrm{M}$ TF for $24 \mathrm{~h}$ and evaluated by flow cytometry as described in Materials and methods. 
Table II. The cytotoxic effect of the combinations of IMPDH inhibitors and ATRA employed simultaneously or sequentially in HL60 cells. ${ }^{\mathrm{a}}$

\begin{tabular}{llcc}
\hline $\begin{array}{l}\text { First } \\
\text { treatment }\end{array}$ & $\begin{array}{c}\text { Second } \\
\text { treatment }\end{array}$ & $\begin{array}{c}\text { \% Cytotoxicity } \\
\text { (mean } \pm \text { S.D.) }\end{array}$ & C.I. \\
\hline Medium & TR & $41 \pm 5$ & - \\
Medium & TF & $43 \pm 6$ & - \\
Medium & ATRA & $18 \pm 4$ & - \\
Medium & TR + ATRA & $51 \pm 7$ & 1 \\
Medium & TF + ATRA & $57 \pm 2$ & 0.9 \\
TR & ATRA & $37 \pm 5$ & 1.3 \\
ATRA & TR & $46 \pm 5$ & 1.1 \\
TF & ATRA & $51 \pm 8$ & 1 \\
ATRA & TF & $47 \pm 5$ & 1.1 \\
\hline
\end{tabular}

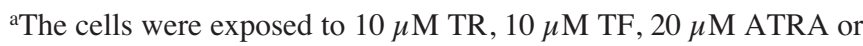
a drug-free medium for $24 \mathrm{~h}$, washed and then exposed to the second drug for a further 24 h. ${ }^{b}$ C.I. indicates the combination index calculated as indicated in Materials and methods. Significant synergism is indicated by a C.I. value of $\leq 0.7$.
Table III. The cytotoxic effect of the combinations of IMPDH inhibitors and MC18 used simultaneously or sequentially in HL60 cells. ${ }^{\mathrm{a}}$

\begin{tabular}{llcc}
\hline $\begin{array}{l}\text { First } \\
\text { treatment }\end{array}$ & $\begin{array}{c}\text { Second } \\
\text { treatment }\end{array}$ & $\begin{array}{c}\text { \% Cytotoxicity } \\
\text { (mean } \pm \text { S.D.) }\end{array}$ & C.I. \\
\hline Medium & TR & $41 \pm 6$ & - \\
Medium & TF & $43 \pm 8$ & - \\
Medium & MC18 & $24 \pm 6$ & - \\
Medium & TR + MC18 & $50 \pm 7$ & 1 \\
Medium & TF + MC18 & $49 \pm 4$ & 1.2 \\
TR & MC18 & $68 \pm 8$ & 0.8 \\
MC18 & TR & $78 \pm 9$ & 0.6 \\
TF & MC18 & $73 \pm 5$ & 0.7 \\
MC18 & TF & $78 \pm 10$ & 0.6 \\
\hline
\end{tabular}

aThe cells were exposed to $10 \mu \mathrm{M}$ TR, $10 \mu \mathrm{M}$ TF, $20 \mu \mathrm{M}$ MC18 or a drug-free medium for $24 \mathrm{~h}$, washed and then exposed to the second drug for a further $24 \mathrm{~h} .{ }^{\mathrm{b}} \mathrm{C}$.I. indicates the combination index calculated as indicated in Materials and methods. Significant synergism is indicated by a C.I. value of $\leq 0.7$.
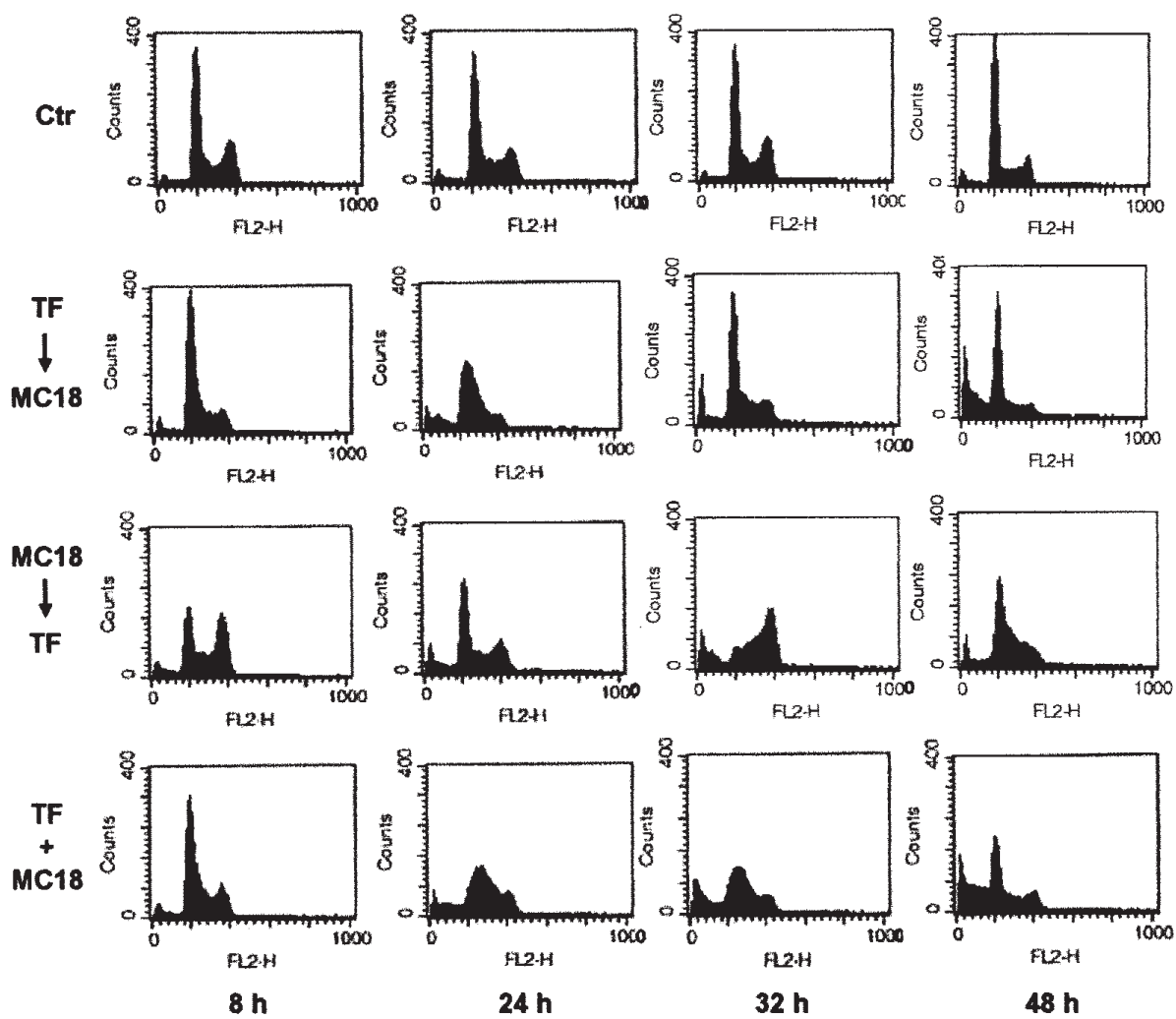

Figure 2. The cell cycle distribution of HL60 cells exposed sequentially or simultaneously to the combination of TF and MC18. In the sequential treatment, the cells were treated with the first drug (TF $10 \mu \mathrm{M}$ or MC18 $0.5 \mu \mathrm{M}$ ) for $24 \mathrm{~h}$ and were then washed and exposed to the second drug for another $24 \mathrm{~h}$. In the simultaneous treatment the cells were treated with the combination and were exposed for $48 \mathrm{~h}$. The cell cycle distribution was determined at $8,24,32$ and $48 \mathrm{~h}$ by flow cytometry.

MC18 is able to elicit significant cytotoxic and apoptotic effects in HL60 cells when employed at concentrations as low as $0.5 \mu \mathrm{M}$ (12). However, unlike other retinoids, this compound induces an unexplained G2-M recruitment after 8-h exposure in HL60 cells. Hence, we exposed HL60 cells for $24 \mathrm{~h}$ to the combinations of different concentrations of MC18 


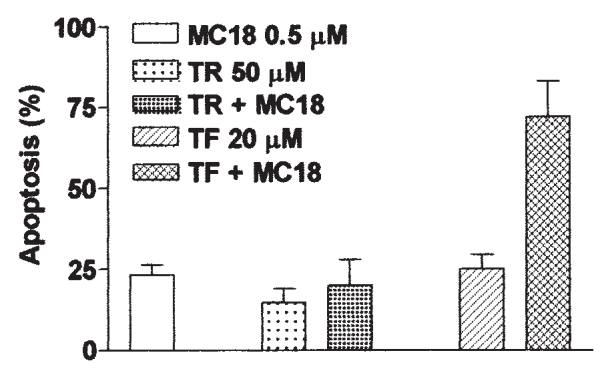

Figure 3. The apoptotic effects induced by the combinations of IMPDH inhibitors and MC18 in HL60 cells. The cells were exposed for $48 \mathrm{~h}$ to the compounds and apoptosis was evaluated by fluorescence microscopy. Results are the mean \pm S.D. of at least three different experiments.

and TF or TR for a comparison. As shown in Table III, the cytotoxic effects of TF in combination with MC18 resulted in additive cytotoxicity, whereas, when TR was employed, the observed effect was slightly antagonistic. Again, we wanted to determine whether this result may be dependent upon the sequence of exposure: interestingly, in the case of both sequential treatments, synergistic effects were observed especially when the retinoid treatment preceded the IMPDH inhibitor.

Combinatory effects on the cell cycle. We wanted to determine whether the differential effects upon cell proliferation were associated with a modification in the cell cycle distribution in HL60 cells. Thus, we treated the cells with the different combinations either simultaneously or sequentially and examined the cell cycle after different times of exposure by flow cytometry (Fig. 2). The results show that in the simultaneous treatment the cells persisted in a block in the $S$ phase whereas those treated sequentially retained the ability to proceed in the cell cycle. However, it is worth noting that a high proportion of cells undergoing apoptosis was evident in the samples treated simultaneously after 48-h exposure.

Combinatory apoptotic effects of TF and MC18. It is well known that most antineoplastic drugs, irrespective of their antiproliferative effects, ultimately trigger cell death by activating the apoptotic process. Hence, we wanted to determine whether a combination of IMPDH inhibitors and retinoids may elicit synergistic apoptotic effects; to this purpose, HL60 cells were treated with MC18 and TF or TR at slightly apoptotic concentrations for $48 \mathrm{~h}$. As shown in

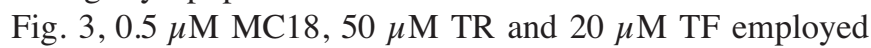
alone were able to induce percentages of apoptotic cells of $23 \%, 15 \%$ and $25 \%$, respectively; instead, the combination of MC18 and TF resulted in $72 \%$ of apoptotic cells, a value that compares favourably with the result obtained by the combination of MC18 and TR (20\% apoptosis), indicating a significant synergistic interaction for TF. Apoptosis is a strictly regulated process involving several biochemical steps including the activation of a series of proteolytic enzymes called caspases proceeding via two main pathways, a death receptor-regulated pathway known as the extrinsic pathway and the intrinsic mitochondrial pathway. Moreover, other alternative pathways either caspase-
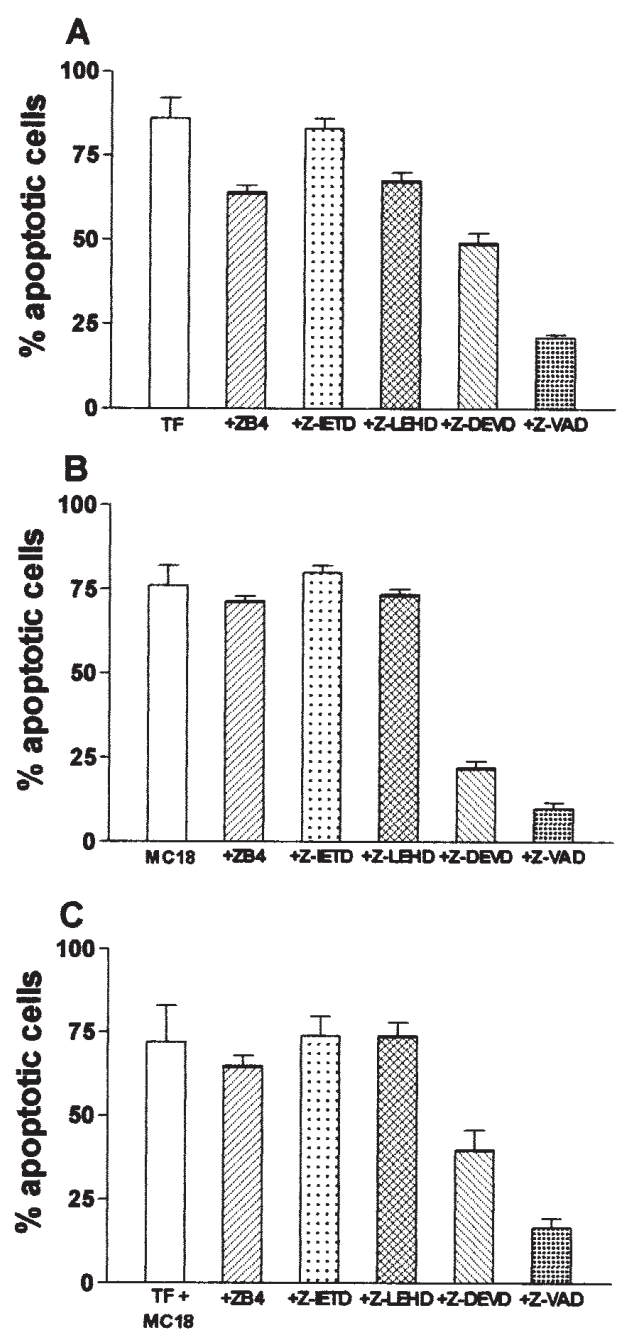

Figure 4. The effects of the anti-Fas antagonistic monoclonal antibody ZB4 and the caspase inhibitors on apoptosis induced by TF (A), MC18 (B) or the combination of TF + MC18 (C) in HL60 cells. The cells were exposed to the inhibitors for $2 \mathrm{~h}$ and treated with $50 \mu \mathrm{M} \mathrm{TF}, 1 \mu \mathrm{M}$ MC18 or $20 \mu \mathrm{M}$ TF + $0.5 \mu \mathrm{M} \mathrm{MC18}$ for $48 \mathrm{~h}$. Apoptosis was evaluated by fluorescence microscopy. Results are the mean \pm S.D. of at least three different experiments.

dependent or caspase-independent have been described $(19,20)$. To elucidate whether the result obtained by the combination may be the consequence of the activation of different apoptotic pathways, we treated the cells with the compounds for $48 \mathrm{~h}$ in the presence of several inhibitors of the intrinsic or extrinsic apoptotic pathways. As shown in Fig. 4, TF-induced apoptosis was inhibited significantly in the presence of the pancaspase inhibitor Z-VAD; moreover, it was inhibited only partially by the caspase 9 and 3 inhibitors and was not affected by the anti-Fas antibody or the caspase 8 inhibitor, suggesting that the apoptotic process was caspase-dependent partially proceeding via the intrinsic pathway. Notably, this conclusion was also supported by the observation that the compounds were able to induce apoptosis in HuT78B1 cells, a cell line resistant to Fastriggered apoptosis due to the expression of a mutated truncated form of the Fas receptor (data not shown). Instead, the retinoid MC18 activated a caspase 3-dependent pathway. Likewise, when the cells were treated with the combination of TF and MC18 the apoptotic process implicated caspase 3 

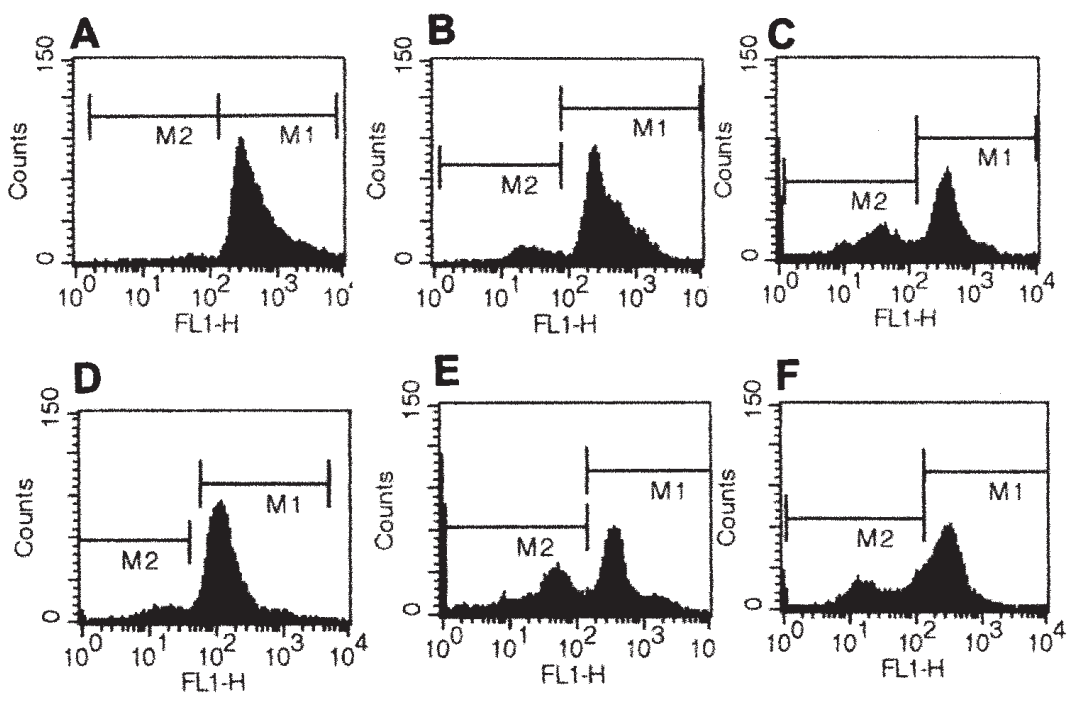

\begin{tabular}{llcc}
\hline & & M1 & M2 \\
& & (\% Normal $\Delta \Psi)$ & (\% Disrupted $\Delta \Psi$ ) \\
\hline A & Control & 96 & 4 \\
B & TF $20 \mu M$ & 90 & 10 \\
C & TF $50 \mu M$ & 63 & 37 \\
D & MC18 $0.5 \mu M$ & 90 & 10 \\
E & MC18 $1.0 \mu M$ & 56 & 44 \\
F & TF $20 \mu M+$ MC18 $0.5 \mu M$ & 76 & 24 \\
\hline
\end{tabular}

Figure 5. The effects of TF and MC18 alone or in combination on the mitochondrial membrane potential ( $\Delta \Psi)$. HL60 cells were treated with drugs for $24 \mathrm{~h}$ and then stained with $\mathrm{DiOC}_{6}$. Fluorescence was evaluated by flow cytometry. The M1 and M2 gates demarcate the cell populations with a normal or disrupted $\Delta \Psi$, respectively. Corresponding numeric values are shown in the Table.

activation without a clear involvement of the classical extrinsic or intrinsic pathways.

Effect of the combination on the mitochondrial function in HL60 cells. Mitochondria play a major role in the induction of apoptosis by cytotoxic drugs. Indeed, lethal damage induces a disruption of the normal mitochondrial membrane potential leading to the release of cytochrome $\mathrm{C}$ and other pro-apoptotic proteins capable of activating the executioner caspases.

Hence, in order to explore the role of mitochondria in the triggering of the apoptotic process by $\mathrm{TF}$ and MC18, we also studied the variations in the mitochondrial membrane potential after 24-h exposure of HL60 cells to the drugs. As shown in Fig. 5, the results show a concentration-dependent disruption of $\Delta \Psi$ by both compounds used alone as well as a proportional reduction of $\Delta \Psi$ in the cells treated with the combination.

\section{Discussion}

The combination of different chemotherapeutic agents has long been considered the most valuable strategy in cancer therapy. Indeed, most successful anticancer regimens, since the introduction of MOPP in lymphoma treatment in the early 70s and till the recently approved Idarubicin + ATRA combination in human prolymphocytic leukaemia, support this notion. Attacking different molecular targets with an effect upon several phases of the cell cycle together with a different spectrum of side-effects are the key determinants of this success. However, the emergence of drug resistance is a strong limitation of classical chemotherapy and novel biological agents such as retinoids drew attention due to their ability to target the non-proliferative drug-insensitive G1 cells (21). Furthermore, the new class of IMPDH inhibitors have been reported to affect signal transduction with significant effects on cell growth and apoptosis. In this study we tested the hypothesis that the combination of the IMPDH inhibitor $\mathrm{TF}$ and retinoids may elicit synergistic antitumor effects in vitro. TF, as well as the reference compound TR, unlike other G1-specific IMPDH inhibitors, have been shown to block the cells in the $S$ phase, suggesting that distinct mechanisms of action may be operative for the different compounds $(4,15)$. Furthermore, the ability of the drug to elicit comparable antiproliferative effects in multidrug-resistant cells supports its possible usefulness in the treatment of Pglycoprotein-expressing tumors.

Hence, we tested the combination of $\mathrm{TF}$ and retinoids in HL60 cells. Provided the two classes of drugs were able to act on different phases of the cell cycle, the cells were treated either contemporaneously or sequentially with the aim of maximizing the possible interaction. The results show that, when ATRA was employed as the retinoid, the association was not particularly advantageous with an effect in the range of additivity. This result was somewhat expected due to the ability of ATRA to promote differentiation, resulting in reduced proliferation and a reduced sensitivity to cytotoxic drugs such as the $\mathrm{S}$ phase-specific TF. The additive effect observed, therefore, could most probably be ascribed to an action on the different cellular populations with different levels of sensitivity to the two drugs. On the contrary, synergistic effects were observed when TF was associated 
with the atypical retinoid MC18. Although this molecule has been shown to bind and efficiently transactivate the retinoid receptors, its antiproliferative and apoptotic effects are receptor independent, and are evident at concentrations significantly lower than those capable of activating the receptors (12). Notably, synergism was evident only in the case of the sequential treatment as far as the antiproliferative effect is concerned. Indeed, the cytofluorimetric assay showed that MC18 has the ability to transiently block tumor cells in the $\mathrm{G}_{2} \mathrm{M}$ phase of the cell cycle, being devoid of any significant differentiating effects as previously shown (12). A similar effect on the cell cycle was reported with some RAR $\gamma$-specific retinoids, possibly due to an increase in the cdk2 activity promoting the $\mathrm{G}_{1} \mathrm{~S}$ transition in specific cell lines (22). Thus, the reversible phase-blocking effect of the two tested molecules may well provide a synchronizationlike mechanism that could account for the sensitization to the effects of the second drug in the sequence after the proper interval time. Likewise, Yeh et al reported the synergistic effect of employing TR in a sequential combination with taxanes, agents which are known to induce a $\mathrm{G}_{2} \mathrm{M}$ arrest in the cell cycle (23). However, a molecular effect linked to the biochemical pathways activated by the two drugs may also be the case. We examined the effects of the combination in the context of apoptosis induction.

Although both compounds were clearly able to induce apoptosis, we found a significant synergistic increase in the number of apoptotic cells by the combination of the two drugs. This effect appears to be cell cycle-independent, since it was apparent particularly in the wells treated with the drugs simultaneously. We wanted to determine whether this result could be due to the activation of different molecular pathways. However, the experiments carried out with several inhibitors of apoptosis demonstrated that both compounds activate a caspase-dependent process possibly involving the activation of the intrinsic mitochondrial pathway, as suggested by the measurement of the mitochondrial membrane potential. These results are in agreement with those recently reported by others employing the IMPDH inhibitor benzamide riboside (24). Furthermore, apoptosis induced by ATRA or other retinoids can also involve mitochondrial dysfunction $(25,26)$. However, the failure of the caspase 9 inhibitor to suppress apoptosis indicates that an alternative pathway may be involved. Indeed, the pancaspase inhibitor Z-VAD was able to inhibit apoptosis induced by the combination. Hence, the potentiation of the apoptotic effects were not due to the activation of different pathways but rather due to a 'priming' facilitating mechanism that accelerates apoptosis induction by a single pathway.

In conclusion, the data reported in this article support the idea that a combination of an IMPDH inhibitor and retinoids may find clinical application in the treatment of leukaemia, especially in retinoid and drug-resistant patients. In vivo studies are needed to evaluate TF and MC18 from a toxicological point of view both alone and in combination before any possible human application.

\section{Acknowledgements}

The research was supported by the Italian MIUR (PRIN 2004).

\section{References}

1. Tricot G and Weber G: Biochemically targeted therapy of refractory leukaemia and myeloid blast crisis of chronic granulocytic leukaemia with tiazofurin, a selective blocker of inosine 5'-phosphate dehydrogenase activity. Anticancer Res 16: 3341-3347, 1996.

2. Kharbanda SM, Sherman ML and Kufe DW: Effects of tiazofurin on guanine nucleotide binding regulatory proteins in HL-60 cells. Blood 75: 583-588, 1990.

3. Weber G, Shen F, Orban TI, Kokeny S and Olah E: Targeting signal transduction. Adv Enzyme Regul 43: 47-56, 2003.

4. Szekeres T, Fritzer M, Pillwein K, Felzmann T and Chiba P: Cell cycle dependent regulation of IMP dehydrogenase activity and effect of tiazofurin. Life Sci 51: 1309-1315, 1992.

5. Jayaram HN, Grusch M, Cooney DA and Krupitza G: Consequences of IMP dehydrogenase inhibition, and its relationship to cancer and apoptosis. Curr Med Chem 6: 561-574, 1999.

6. Yalowitz JA, Pankiewicz K, Patterson SE and Jayaram HN: Cytotoxicity and cellular differentiation activity of methylenebis(phosphonate) analogs of tiazofurin and mycophenolic acid adenine dinucleotide in human cancer cell lines. Cancer Lett 181: 31-38, 2002.

7. Mandelli F, Diverio D, Avvisati G, et al: Molecular remission in PML/RAR alpha-positive acute promyelocytic leukemia by combined all-transretinoic acid and idarubicin (AIDA) therapy. Gruppo Italiano-Malattie ematologiche maligne dell'adulto and Associazione Italiana di ematologia ed Oncologia pediatrica Cooperative Groups. Blood 90: 1014-1021, 1997.

8. Altucci L, Wilhelm E and Gronemeyer H: Leukemia: beneficial actions of retinoids and rexinoids. Int $\mathrm{J}$ Biochem Cell Biol 36: 178-182, 2004.

9. Ortiz MA, Bayon Y, Lopez-Hernandez FJ and Piedrafita FJ: Retinoids in combination therapies for the treatment of cancer: mechanisms and perspectives. Drug Resist Updat 5: 162-175, 2002.

10. Simoni D, Invidiata FP, Rondanin R, et al: Structure-activity relationship studies of novel heteroretinoids: induction of apoptosis in the HL60 cell line by a novel isoxazole-containing heteroretinoid. J Med Chem 42: 4961-4969, 1999.

11. Simoni D, Roberti M, Invidiata FP, et al: Programmed cell death (PCD)-associated with the stilbene motif of arotinoids: discovery of novel apoptosis inducer agents possessing activity on multidrug resistant tumor cells. Bioorg Med Chem Lett 10: 2669-2673, 2000.

12. Simoni D, Roberti M, Invidiata FP, et al: Heterocyclecontaining retinoids. Discovery of a novel Isoxazole Arotinoid possessing potent apoptotic activity in multidrug and druginduced apoptosis resistant cells. J Med Chem 44: 2308-2318, 2001.

13. Franchetti P, Cappellacci L, Grifantini M, Barzi A, Nocentini G and O'Connor YH: Furanfurin and thiophenfurin: two novel tiazofurin analogues. Synthesis, structure, antitumor activity, and interactions with inosine monophosphate dehydrogenase. J Med Chem 38: 3829-3837, 1995.

14. Franchetti P, Cappellacci L, Perlini P, et al: Isosteric analogues of nicotinamide adenine dinucleotide derived from furanfurin, thiophenfurin, and selenophenfurin as mammalian inosine monophosphate dehydrogenase (Type I and II) inhibitors. J Med Chem 41: 1702-1707, 1998.

15. Laliberté J, Yee A, Xiong Y and Mitchell BS: Effects of guanine nucleotide depletion on cell cycle progression in human T lymphocytes. Blood 91: 2896-2904, 1998.

16. Webb JL: Effect of more than one inhibitor. In: Enzymes and metabolic inhibitors. Academic Press, New York, pp66-79, 1966.

17. Duke RC and Cohen JJ: Morphological and biochemical assays of apoptosis. In: Current Protocols in Immunology. Coligan JE and Kruisbeak AM (eds). John Wiley \& Sons, New York, pp3.17.1, 1992.

18. Darzynkiewicz Z, Bruno S, Del Bino G, et al: Features of apoptotic cells measured by flow cytometry. Cytometry 13: 795-798, 1992.

19. Wang Z-B, Liu Y-Q and Cui Y-F: Pathways to caspase activation. Cell Biol Int 29: 489-496, 2005.

20. Abraham MC and Shaham S: Death without caspases, caspases without death. Trends Cell Biol 14: 184-193, 2004.

21. Simoni D and Tolomeo M: Retinoids, apoptosis and cancer. Curr Pharm Des 7: 1821-1835, 2001. 
22. Shyu RY, Lin DY, Reichert U and Jiang SY: Synthetic retinoid CD437 induces cell-dependent cycle arrest by differential regulation of cell cycle associated proteins. Anticancer Res 22: 2757-2764, 2002.

23. Yeh YA, Olah E, Wendel JJ, Sledge GW and Weber G: Synergistic action of taxol with tiazofurin and methotrexate in human breast cancer cells: schedule dependence. Life Sci 24: 431-435, 1994.

24. Khanna N, Jayaram HN and Singh N: Benzamide riboside induced mitochondrial mediated apoptosis in human lung cancer H520 cells. Life Sci 75: 179-190, 2004.
25. Ortiz MA, Lopez-Hernandez FJ, Bayon Y, Pfahl M and Piedrafita FJ: Retinoid-related molecules induce cytochrome $c$ realease and apoptosis through activation of c-Jun $\mathrm{NH}(2)-$ terminal kinase/p38 mitogen-activated protein kinases. Cancer Res 61: 8504-8512, 2001.

26. Zheng A, Mantymaa P, Saily M, Siitonen T, Savolainen ER and Koistinen P: An association between mitochondrial function and all-transretinoic acid-induced apoptosis in acute myeloblastic leukaemia cells. Br J Haematol 105: 215-224, 1999. 